\title{
Implementing a three-tiered service model for knowledge syntheses at an academic teaching hospital
}

\author{
Linda Yang, Ani Orchanian-Cheff ${ }^{1}$, Melanie Anderson, Ashley Farrell, Tim Tripp
}

\begin{abstract}
Introduction: In response to increasing demand for library support, and problems observed in working with researchers new to knowledge syntheses, University Health Network (UHN) Library and Information Services designed and implemented a new Knowledge Synthesis (KS) Service model to meet the demand while providing an opportunity to educate researchers about KS methodological guidelines and reporting standards. Description: The new service model includes three tiers: consultation only, support for course work and dissertations, and full-service with librarian co-authorship. It is accompanied by more robust information on the library's website about synthesis methods. Outcomes: An inductive thematic analysis was performed on transcripts of interviews with the seven Information Specialists to determine their experience with the new service model. Eight themes emerged: workload, control/agency, communication, professional recognition, client knowledge, client attitudes/expectations, quality, and concern over buy-in. The new service model was generally well received. Discussion: UHN Libraries could not directly adopt existing tiered models without changes to account for students in the hospital working on syntheses for reasons other than coursework. Tiers based on those found in an environmental scan were adapted for clientele who perform multiple roles. The impact on researcher experience, publication rates, and Information Specialists' workload will be examined in the future when more data are available.
\end{abstract}

\section{Introduction}

The publication of systematic reviews (SRs) is increasing; unfortunately, many have added to research waste through poor methods and reporting [1]. This increase has been reflected in research activity at University Health Network (UHN); in 2018 alone, there were 138 requests for new SRs received by seven information specialists (ISs). Requests were also received for types of knowledge syntheses (KS) such as scoping reviews and rapid reviews, for which the existing services were not tailored. The amount of time ISs spent supporting SRs increased four-fold over six years (2012 to 2018) (Figure 1).

Conducting these searches was often made difficult because of researchers who did not have a project team, had allocated insufficient time to complete the project, did not have a review protocol, or were unfamiliar with
SR methodology. Similar patterns have been observed by other libraries $[2,3]$. One consequence was that projects were abandoned after an IS had already expended time developing and executing complex search strategies. When projects were completed, the ISs were not consistently being included as authors of their work. Further, some researchers misrepresented the ISs' work by writing the manuscript without accepting IS input. Many of the challenges that ISs experienced have also been described by Nicholson, McCrillis, and Williams [4].

A new model was designed and implemented with the intention to provide KS services to meet client demand while providing an opportunity to educate clients about KS methodological guidelines and reporting standards, as well as provide a mechanism for ISs to diplomatically require authorship or withdraw from projects not meeting standards. The IS team decided that a tiered KS service model should be

Linda Yang, Faculty of Information, University of Toronto

Ani Orchanian-Cheff ${ }^{1}$, Melanie Anderson, Ashley Farrell, Tim Tripp.

Library and Information Services, University Health Network, Toronto, ON, Canada

${ }^{1}$ Corresponding author e-mail: ani.orchanian-cheff@uhn.ca

This article has been peer-reviewed.

(c) Yang, Orchanian-Cheff, Anderson, Farrell, Tripp.

This article is distributed under a Creative Commons Attribution License: https://creativecommons.org/licenses/by/4.0/ 
implemented, allowing clients to choose the level of IS involvement. This approach was based on a 2018 CHLA/ABSC paper presentation from Neilson et al. [5], at the University of Manitoba (UM), where librarians conducted an environmental scan of SR support models from libraries across North America, the UK, and Australia, and decided to implement a three-tiered model: instruction, consultation, and research and publication support. UHN Libraries modified the three-tiered service approach to fit the needs of a client population in an academic hospital setting. The ISs modified UM's consultation tier because it was focused on a specific population (UM graduate students, residents, and faculty), while UHN Libraries wanted to create services that would offer both consultation and instruction to any staff member or learner. This would include any medical, nursing or allied health students or clinicians who needed guidance. Researchers and learners who are affiliated with UHN but are conducting a knowledge synthesis as part of the coursework or thesis for an academic institution also used UHN Libraries.

\section{Program Description}

UHN Libraries decided to offer three tiers of KS services to UHN staff, learners, and researchers. The first tier (consultation only) is for researchers who would like to receive advice, instruction, or assistance on a variety of KS-related topics during one or more meetings, limited to one hour in length, at the discretion of the IS. This tier would not involve the IS in search strategy development or any other tasks that would warrant co-authorship. A pre-existing protocol would not be required.

It was important to direct researchers performing coursework into a distinct tier in order to steer them into a more appropriate service for their needs. This was a marked change from the previous services, in which the assumption was that KS projects by UHN affiliated researchers were work related. Thus, ISs occasionally discovered that they had conducted searches submitted as part of graded assignments or significant coursework. In this new second tier, an IS assists UHN researchers or students completing coursework, dissertations, or theses with choosing the KS method, developing the research question, finding guidelines and reporting standards, choosing databases, and selecting and using a citation manager. The IS can also provide advice on appropriate search methods, search strategies, dissemination of a published paper, and grey literature searching. Once again, the IS would not be directly responsible for creating and executing the search strategies or any other tasks that would warrant co-authorship. This tier is distinct from the first in that, at their discretion, the IS provides a greater number of consultations, including more detailed search guidance and feedback. The second tier also serves to make services for coursework distinct for clients looking for library involvement in their KS.

At the third tier, the full service model, ISs are included as part of the research team and contribute to the KS by developing and executing search strategies in all databases required, documenting search results, conducting cited reference searching, downloading search results, updating or rerunning searches, and writing the search methods and corresponding appendix for the manuscript.

As part of this service change, the IS team agreed to modify the existing SR request form into a KS proposal form for researchers using the third-tier (Appendix 1). To make sure that researchers worked through the planning process and were aware of the resources, time, and work that a KS involves, they would now need to provide a protocol along with the completed proposal form. The proposal form has firm expectations regarding co-authorship in keeping with the organization's Research Authorship Policy and the International Committee of Medical Journal Editors' Recommendations for the Conduct, Reporting, Editing, and Publication of Scholarly Work in Medical Journals $[6,7]$. The "Terms of Service," to be signed by both the IS and the principal investigator, includes a clause that states that the IS has the right of final approval for any version sent for publication, and that UHN Libraries reserves the right to withdraw involvement from any project that does not align with best practices.

The new KS service model [8] was launched on the library's website in February 2019 in conjunction with a page about types of reviews and their methodology [9]. This second page included information about the different standards, reporting guidelines, timelines, and team makeup for traditional reviews and various types of KS such as systematic, scoping, or rapid reviews.

\section{Outcomes}

Time spent on knowledge synthesis projects appears to have been reduced since the current KS service model was initiated in February 2019 (Figure 2); 
however, the data need to be re-evaluated after more time has passed to determine if this trend is real and consistent.

With the launch of this service, UHN Libraries started counting all knowledge syntheses rather than the earlier practice of counting only systematic reviews. In 2019 there were 139 new knowledge synthesis proposals, compared to 138 systematic review requests in 2018. It is likely that, since scoping reviews and other knowledge syntheses are now included, the number of systematic reviews has been reduced. However, these statistics are not directly comparable, and it is too early to draw any serious conclusions.

From February to December 2019, ISs provided 55 KS consultations (both tiers 1 and 2). In the past any KS assistance akin to what is now "consultations" were included in the library's instruction statistics. Since this service was not tracked separately, it is not yet possible to comment on any trends.

To gauge how the changes had impacted their experience and workload, six months after the new service was launched a library and information sciences student (L.Y.) conducted, recorded and transcribed interviews with all seven ISs. The questions asked addressed both their experiences with the old model and the new one (Appendix 2). An inductive thematic analysis [10] was then performed by three of the authors (A.O., M.A., A.F.) independently.

Eight themes emerged: workload, control/agency, communication, professional recognition, client knowledge, client attitudes/expectations, quality, and concern over buy-in. In most cases interviewees' comments referred to the third-tier of service.

All seven interviewees commented on the amount of work involved in providing knowledge synthesis services to their clients. Many also commented about the positive effect of a reduced workload when it came to these requests and the ability to channel that time into other projects. As one interviewee noted, "It's nice to be working on two reviews instead of nine."

Six interviewees felt that before the change in services they lacked control in areas including which projects to work on, how their work was represented in publications, and the quality of the projects. Three interviewees also mentioned that before the change they had little control over client demands, especially when clients were trying to cut corners. After the change in service models ISs felt that they had more control over their work by leveraging authorship: "This gives me more control over which projects I invest time in because I can say if you want me to do these for you, you need to provide me with these things."

Three ISs felt that the changes to the website enabled more formalized communication with clients about what services are offered. The proposal form was lauded by six ISs for providing clear communication between the client and IS, helping with difficult conversations, preventing miscommunication, and providing a framework for meeting with clients. "I do like having the knowledge synthesis project proposal; it gives me something to anchor everything to, and I can always go back and say this is what we talked about."

A lack of recognition was mentioned by six interviewees as an important stressor, because granting authorship was previously a choice for the client and not something guaranteed. ISs felt that they were putting in a lot of work that was not being recognized; there was a feeling of being disrespected. "The amount of work we'd done didn't get appreciated and...the research team said we'll put you as co-author, but they didn't... that's our biggest issue, for our work to be known, acknowledged properly."

All of the ISs reflected on client knowledge or lack of knowledge noting that clients were not familiar with what a systematic review was or the amount of work such a project entailed. "So a lot of times, they're thinking just a simple literature search, but they'll come and say they want to do a systematic review." Previously, clients were still learning about systematic review methodology throughout their projects, which increased the time investment as work needed to be repeatedly revised. The new service model, however, ensured that clients would learn about the review method before embarking on the project.

Five ISs commented that before the change clients had unrealistic expectations regarding the amount of work required and felt that there should be a quick turnaround on search requests: "the expectation they have is they're going to get all the systematic review searches done... the librarian's going to get them all done by next week because they're doing a systematic review in a month, which is completely unreasonable." ISs felt that when clients were writing a protocol and planning the steps that needed to be taken, they were less surprised at the amount of work involved.

Six interviewees reflected on the issue of quality. The ISs stated that clients would push to take shortcuts that would sacrifice the quality of a systematic review and at times go against review standards. "You're doing work for these projects where people are trying to take shortcuts right away and you try to talk them out of it 
but you're still working on this project that you know it isn't going to end up as something good." But when the new service model was instituted it was felt that the projects " $[. .$.$] that are making their way through all of$ the hurdles are the ones that are actually of quality, and the people are demonstrating that they're going to be able to put the work in.”

Finally, there was concern from six ISs that these changes would result in pushback from clients not willing to write a protocol, or that clients would not be willing to provide co-authorship for the work that was provided: "[...] there is worry about saying that we have to be co-authors, that clients aren't going to want to give us co-authorship or aren't going to want to work with us... you don't want to alienate people.” ISs were not clear if this was a real trend, and if it was, whether it was a problem.

\section{Discussion:}

In response to an increase in requests for IS involvement in KS projects and observed lack of preparedness by researchers in engaging in such projects, UHN Libraries changed their service model from "one-size-fits-all” to a three-tiered model that was generally well received by ISs in the first six months.

Much of the literature regarding structured systematic review services is from academic libraries [2,12-15] that provide services based on the requester's role, such as students or faculty. However, students in a hospital might be working on systematic reviews as part of their work at the hospital separate from their coursework. UHN Libraries, therefore, needed to provide service to these students and simultaneously provide a separate level of service to people working on coursework. Consequently, one marked change is our offering a distinct tier for those completing coursework, providing learners specialized and expanded consultations while they complete their academic requirements. Students are also eligible for any tier of service depending on the purpose of their project. This approach can be applied in other institutions serving clientele fulfilling multiple roles.

One of the aims of establishing different tiers of service was reducing the time ISs spend on projects that do not have client commitment, thereby freeing them for work on projects more likely to reach completion. This aim will be examined with data collected at the two- and five-year marks to allow for time to publication. The impact on researcher experience, publication rates, and IS workload will also be examined in the future when more data are available.

The exposure to KS standards and the planning required for the protocol could improve the quality of the resulting manuscripts. This would need to be evaluated in the future after KSs have been published under the current service model. The next steps also include evaluating the clients' experience with using the KS service and determining if clients have been following through on their agreement to grant librarian co-authorship.

\section{Statement of Competing Interests}

No competing interests declared.

\section{References}

1. Page MJ, Shamseer L, Altman DG, Tetzlaff J, Sampson M, Tricco AC, et al. Epidemiology and reporting characteristics of systematic reviews of biomedical research: a crosssectional study. PLOS Med. 2016;13(5):e1002028. doi: 10.1371/journal.pmed.1002028.

2. Campbell S, Dorgan M. What to do when everyone wants you to collaborate: managing the demand for library support in systematic review searching. J Can Health Libr Assoc. 2015;36(1)11-9. doi: 10.5596/c15-005.

3. Sikora L, Bass M. Sharpening the other side of the dual edged systematic review sword: expecting more from our users. Paper presented at: CHLA/ABSC 2018. 2018, Jun 15-18; St. John's, Newfoundland.

4. Nicholson J, McCrillis A, Williams JD. Collaboration challenges in systematic reviews: a survey of health sciences librarians. J Med Libr Assoc 2017;105(4):385-93. doi: dx.doi.org/105195/jmla.2017.176.

5. Neilson C, Askin N, Demczuk L, Gottschalk $\mathrm{T}$, Szwajcer A. An environmental scan of systematic review service models. Canadian Health Libraries Association/Association des bibliothèques de la santé du Canada conference; 2018 Jun 15-18; St. John’s, NL.

6. Research Risk \& Audit Committee. Research: research authorship. Policies \& Procedures 
Manual [policy], University Health Network; 2017. (Unpublished report).

7. International Committee of Medical Journal Editors Recommendations [Internet]. International Committee of Medical Journal Editors; c2019. Recommendations for the conduct, reporting, editing, and publication of scholarly work in medical journals; 2018 Dec [cited 2019 Oct 16]; [full document].

Available from: http://www.icmje.org/icmjerecommendations.pdf

8. UHN Virtual Library [Internet]. Toronto: UHN Libraries; c2019. Types of literature reviews; 2019 Oct 2 [cited 2019 Oct 16]; [full page]. Available from:

https://guides.hsict.library.utoronto.ca/c.php?g $=696870 \& \mathrm{p}=5020041$

9. UHN Virtual Library [Internet]. Toronto: UHN Libraries; c2019. Knowledge synthesis services; 2019 Oct 2 [cited 2019 Oct 16]; [full page]. Available from:

https://guides.hsict.library.utoronto.ca/uhnvirt ual library/KSS

10. Braun V, Clarke V. Using thematic analysis in psychology. Qual Res Psychol. 2006;3(2):77101.

11. Ludeman E, Downton K, Shipper AG, Fu Y. Developing a library systematic review service: a case study. Med Ref Serv Q. 2015;34(2):173-80.

12. Knehans A, Dell E, Robinson C. Starting a fee-based systematic review service. Med Ref Serv Q. 2016;35(3):266-73.

13. McKeown S, Ross-White A. Building capacity for librarian support and addressing collaboration challenges by formalizing library systematic review services. J Med Libr Assoc. 2019;107(3):411-9.

14. Riegelman A, Kocher M. A model for developing and implementing a systematic review service for disciplines outside of the health sciences. RUSQ. 2018 Fall 2018;58(1):22-7.

15. Roth SC. Transforming the systematic review service: a team-based model to support the educational needs of researchers. J Med Libr Assoc. 2018;106(4):514-20 
Yang, Orchanian-Cheff, Anderson, Farrell, Tripp

Fig. 1: Hours spent on SRs per year before service model change.

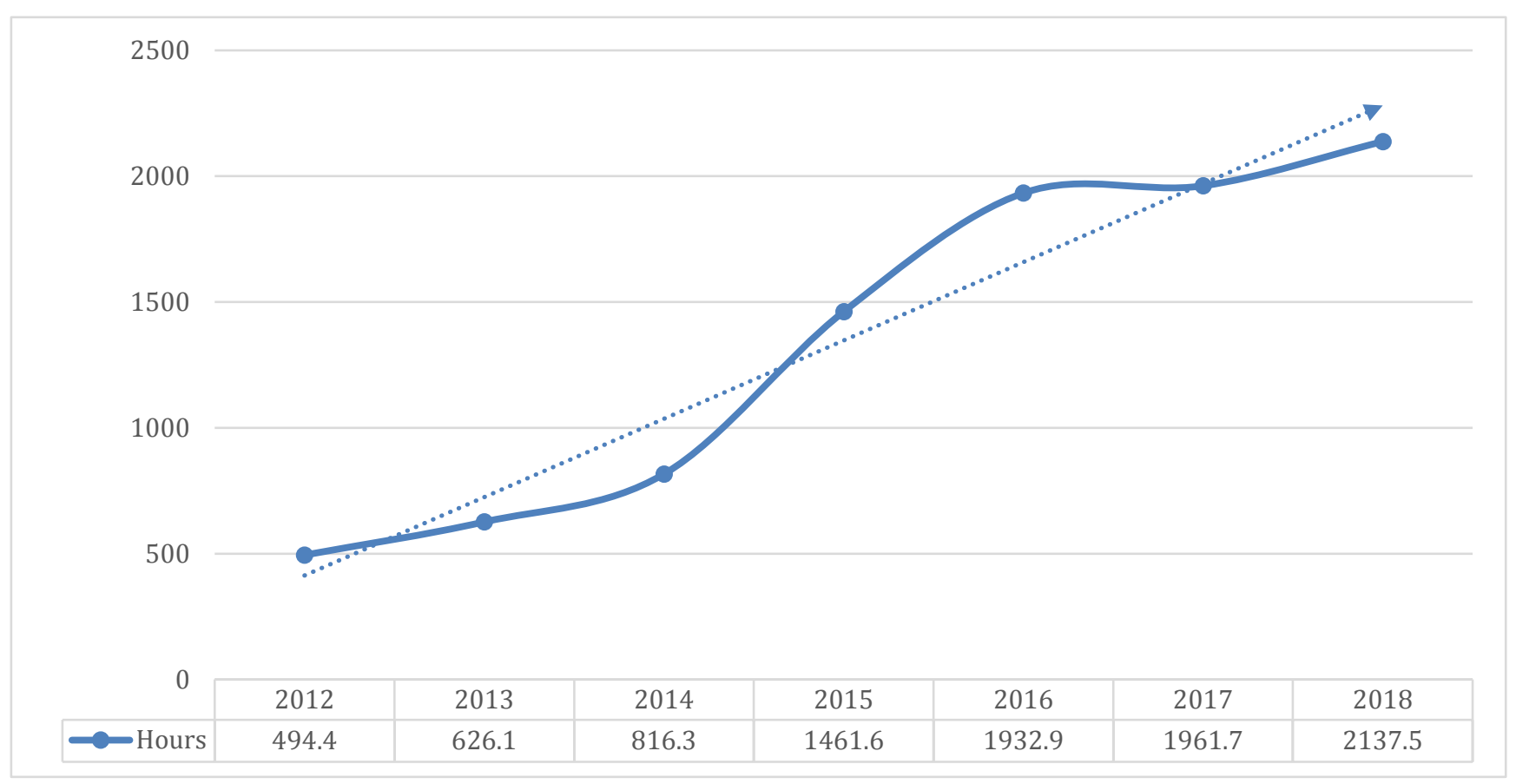


Yang, Orchanian-Cheff, Anderson, Farrell, Tripp

Fig. 2: Hours spent on tier-3 projects per month.

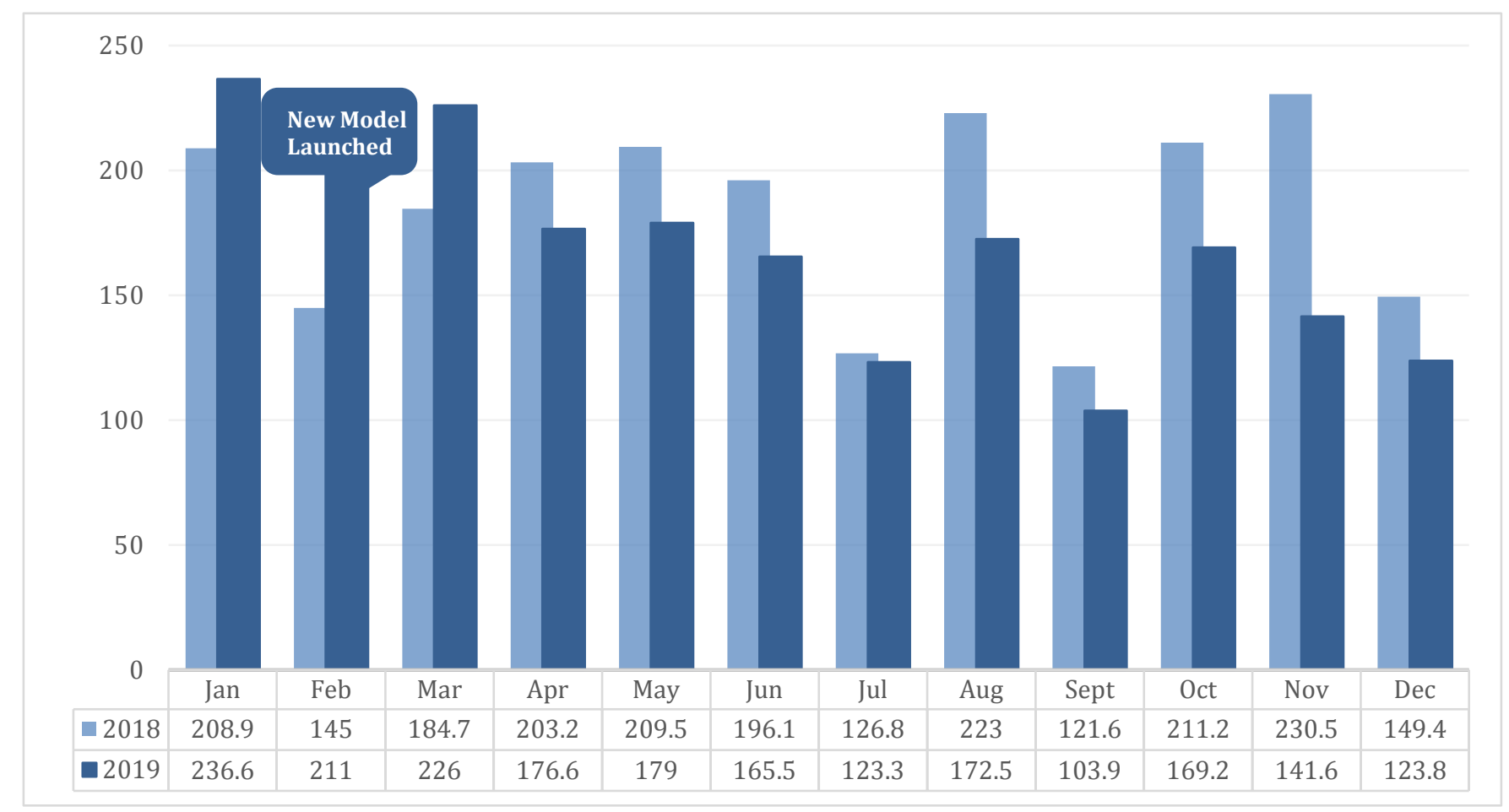


Yang, Orchanian-Cheff, Anderson, Farrell, Tripp

Appendix 1: KS proposal forms

for latest version see https://guides.hsict.library.utoronto.ca/ld.php?content id=34390307

\section{Knowledge Synthesis Project Proposal Form}

To be discussed with an Information Specialist

(1) Before you start:

For systematic reviews, please familiarize yourself with:

- $\quad$ Systematic Review Overview

- PRISMA Checklist

For scoping reviews, please familiarize yourself with:

- Hilary Arksey \& Lisa O'Malley (2007) Scoping studies: towards a methodological framework, International Journal of Social Research Methodology, 8:1, 19-32, DOI: 10.1080/1364557032000119616

(2) Please complete Parts 1 and 2 of this form before meeting with an information specialist (IS).

(3) Please include your review protocol with this form.

\section{PART 1: General Information}

First meeting date:

\section{Timelines}

Initial searches for a systematic or scoping review take 6-8 weeks at minimum. On average, a systematic review project takes a year. If your timelines are shorter, you may wish to consider other types of synthesis projects or a traditional (narrative) review. See suggested timelines for a Cochrane Review for reference.

\section{Contact information}

Principal Investigator:

Name:

Department:

Title:

Email:

Site / Room:

Ext:

If the Principal Investigator will not be interacting with the Information Specialist directly, please indicate the Principal Investigator's representative:

Name:

Email:

Ext:

Information Specialist: 
Yang, Orchanian-Cheff, Anderson, Farrell, Tripp

Name:

Email:

Ext:

Review teams should include content and methodology experts and have access to statistical experience as required. Please list names and affiliations of the other members of the review team:

Are any team members affiliated with the University of Toronto? $\square$ Yes $\square$ No

\section{Type of Review or Synthesis (please see Glossary of Terms)}

Systematic Review $\square$ Scoping Review $\square$ Rapid Review $\square$ Other:

\section{Research Output}

Paper $\square$ Practice Guideline $\square$ Poster $\square$ Other:

\section{Funding}

Have you applied for, or do you plan to apply for, a grant? $\square$ Yes $\square$ No

Grant application to be submitted to: $\square$ CIHR $\square$ Other:

Have you received any other funding for this project? $\square$ Yes $\square$ No

If yes, please specify:

\section{Which citation software will you use for this project?}

Endnote $\square$ Other:

\section{Review Topic}

Describe in a few words the subject of your Review

\section{Research Objectives}

Precise statement of the primary objective, ideally in a single sentence. For example: 'To assess the effects of [intervention or comparison] for [health problem] in [types of people, disease or problem and setting if specified]'. This might be followed by one or more secondary objectives. 
Yang, Orchanian-Cheff, Anderson, Farrell, Tripp

\section{Why is it important to do this review?}

\section{If you plan to submit a manuscript for publication, please list 2-3 journals to which you intend to submit.}

Not all journals will accept systematic reviews or scoping reviews. Some will only publish invited reviews. Some open access journals can also be predatory. It is helpful to be aware of instructions for authors and policies of journals in advance.

\section{Review Protocol and Registration}

For systematic reviews and meta-analysis, we strongly recommend using the PRISMA-P checklist when drafting your review protocol. Some journals (eg. BMJ Open) will expect you to submit a completed checklist with your submission and recommend Prospero for registration of systematic reviews.

Have you already, or do you plan to, register your research protocol? $\square$ Yes $\square$ No

If you have already registered your protocol, please indicate where it can be accessed (e.g., Web address), and, if available, provide registration information including registration number

\section{Ensure that your project does not duplicate any work already published or registered}

Are you aware of any existing systematic or scoping reviews on your topic? $\quad \square$ Yes $\square$ No Sources you searched:
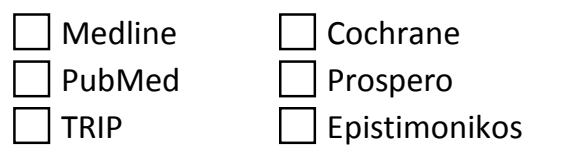

$\square$ Campbell Collaboration $\square$ Joanna Briggs Institute Other:

Search details or search terms used:

\section{Provide citations for relevant systematic or scoping reviews (up to 10).}

- Please provide below the DOI (Digital Object Identifier) of the article or the PMID (PubMed identifier) or list: First author. Article title. Journal title. Vol. Issue. Pages. Year.

- If you have them, please bring papers to the meeting with your IS. 

1.
2.
3.
4.
5.
6.
7.
8.
9.
10.

Please provide information on previous searches for original research articles (i.e. not systematic or scoping reviews) on your topic.

Do you have relevant primary studies (target articles) for your SR?

Target articles are used as a validation set to test potential search strategies. These articles should be ones that absolutely meet your inclusion criteria.

$\square$ Yes $\square$ No

$\begin{array}{ll}\text { Sources you searched: } & \square \text { MEDLINE } \\ & \square \text { PsycINFO } \quad \square \text { PubMed } \\ & \square \text { CINAHL } \quad \square \text { EMBASE } \quad \square \text { other: }\end{array}$

Search details or comments:

\section{Provide citations for target articles (up to 10).}

- Please provide below the DOI (Digital Object Identifier) of the article or the PMID (PubMed identifier) or list: First author. Article title. Journal title. Vol. Issue. Pages. Year.

- If you have them, please bring papers to the meeting with your IS.

1.

2.

3.

4.

5.

6.

7.

8.

9.

10.

\section{PART 2: Defining Your Research Question and Studies to Include}


Yang, Orchanian-Cheff, Anderson, Farrell, Tripp

\section{Structured question (Criteria for considering studies for this review):}

- Identify your criteria and provide synonyms.

- Some review questions may not fit exactly in the PICO frame and not all components may be relevant to your question.

\begin{tabular}{|c|c|c|c|c|}
\hline \multicolumn{5}{|l|}{ Population } \\
\hline \multicolumn{5}{|l|}{ Intervention } \\
\hline \multicolumn{5}{|l|}{$\begin{array}{l}\text { Comparison } \\
\text { (if applicable) }\end{array}$} \\
\hline \multicolumn{5}{|l|}{ Outcomes } \\
\hline \multicolumn{5}{|c|}{$\begin{array}{l}\text { The type of question should dictate the type of study. Listed in the row below are suggested best levels of } \\
\text { evidence for each type of question. }\end{array}$} \\
\hline Type of Question & $\begin{array}{l}\square \text { Therapy or } \\
\text { Therapeutic } \\
\text { Intervention or } \\
\text { Prevention }\end{array}$ & $\begin{array}{l}\square \text { Diagnosis or } \\
\text { Screening }\end{array}$ & $\square$ Etiology/Harm & $\square$ Prognosis \\
\hline Type of Study & $\begin{array}{l}\square \text { Randomized } \\
\text { Controlled Trials } \\
\square \text { Systematic } \\
\text { Review / Meta- } \\
\text { Analysis } \\
\square \text { Controlled } \\
\text { Studies } \\
\square \text { Cohort Studies } \\
\square \text { Case Control } \\
\text { Studies } \\
\square \text { Case Series/Case } \\
\text { reports }\end{array}$ & $\begin{array}{l}\square \text { Randomized } \\
\text { Controlled Trials } \\
\square \text { Controlled Trial } \\
\square \text { Prospective/ } \\
\text { Cohort/Longitudinal/ } \\
\text { Follow-up Studies } \\
\square \text { Cross-Sectional } \\
\text { (prevalence) Studies } \\
\square \text { Case Control } \\
\text { Studies } \\
\square \text { Case Series/Case } \\
\text { reports }\end{array}$ & $\begin{array}{l}\square \text { Randomized } \\
\text { Controlled Trials } \\
\square \text { Controlled Trials } \\
\square \text { Cohort Studies } \\
\square \text { Case Control } \\
\text { Studies } \\
\square \text { Case Series/Case } \\
\text { reports }\end{array}$ & $\begin{array}{l}\square \text { Cohort Studies } \\
\square \text { Longitudinal } \\
\text { Studies }\end{array}$ \\
\hline
\end{tabular}

\section{Other types of studies to include?}

Cost-Benefit Analyses
Qualitative Research

Other: 
Yang, Orchanian-Cheff, Anderson, Farrell, Tripp

\section{Comments:}

\section{Apart from journal articles, which publications do you wish to include or exclude?}

Research in progress

Conference proceedings/abstracts

Dissertations/Theses

Books/ book chapters

Other:

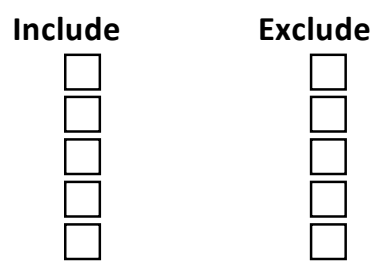

\section{Population}

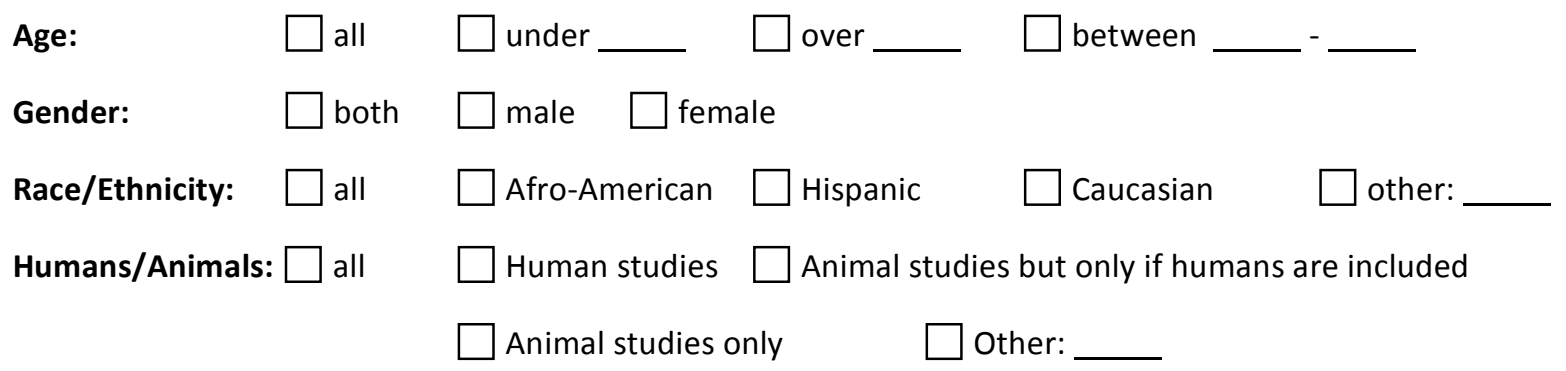

\section{Languages}

It is not advisable to impose any language restrictions on the search.

English Only: $\square$ Yes $\square$ No, all languages

$\square$ No, also include:

\section{Years}

Use date restrictions only when appropriate. For example, drug treatment only available after a certain date.

Years: $\square$ all $\square$ before _ $\square$ after _ $\quad \square$ between

\section{Comments:}


Yang, Orchanian-Cheff, Anderson, Farrell, Tripp

\section{PART 3: During your Meeting with your Information Specialist Databases and Resources Recommended for Search Inclusion}

To be discussed with your Information Specialist

\section{Original research databases}

\section{Medicine}

$\square$ Medline (Ovid)

$\square$ PubMed (non Medline records only)

$\square$ Embase

$\square$ Cochrane Database of Systematic Reviews

$\square$ Cochrane CENTRAL

$\square$ Biosis Previews

$\square$ African Index Medicus

$\square$ LILACS (Latin America)

Other:

\section{Behavioral Sciences and Education}

$\square$ PsycINFO (Ovid)
$\square$ ERIC

\section{Nursing/Allied Health}

$\square$ CINAHL

$\square$ Emcare

$\square$ OT Seeker

$\square$ PsycBite

$\square$ PEDro

$\square$ SpeechBite

\section{Other types}

\section{Citation Indexes}

Use these databases to search for articles that cite or are cited by your eligible articles.

$\square$ Scopus (Elsevier)

$\square$ Web of Science (TR)

Other:

\section{Research in progress}

$\square$ ClinicalTrials.gov

$\square$ International Clinical Trials Registry Platform

$\square$ International Standard Randomised Controlled

Trial Number Registry
Other:

Conference proceedings

$\square$ Biosis Previews

$\square$ Embase

Web of Science

Scopus

Books/Book chapters

\section{Grey Literature}

Literature published outside the traditional publishing ecosystem and not widely disseminated.

CADTH

CADTH Google Custom Search for Canadian HTA sites

Centre for Reviews and Dissemination

CMA Infobase

TRIP Database

National Institute for Clinical Excellence

NIHR Health Technology Assessment Programme

AHRQ - Technology Assessment + EPC Evidence Based

Reports + Research Summaries, Reviews, and Reports

Australia and New Zealand Horizon Scanning Network - Technologies Assessed

$\square$ To identify additional sources: Grey Matters (CADTH tool)

$\square$ OpenGrey (EU)

$\square$ NTIS (US)

$\square$ Google Scholar

$\square$ Other:

\section{Dissertations/Theses}

ProQuest Dissertations and Theses

Other

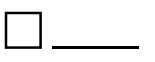


Yang, Orchanian-Cheff, Anderson, Farrell, Tripp

Comments:

JCHLA / JABSC 41: 46-66 (2020) doi: 10.29173/jchla29436 
Yang, Orchanian-Cheff, Anderson, Farrell, Tripp

\section{PART 4: Terms of Service}

\section{(Projects not associated with course work, dissertations or thesis)}

As part of the Knowledge Synthesis Service, an Information Specialist will join your team, create and execute the search strategies, author the search methodology, and be a co-author*. There are no fees associated with the services below.

\begin{tabular}{|c|c|}
\hline & Your Information Specialist will: \\
\hline$\square$ & Check for existing or ongoing systematic or scoping reviews on the same topic \\
\hline$\square$ & Design and run the systematic search strategies in bibliographic databases \\
\hline$\square$ & $\begin{array}{l}\text { Send a copy of the first } 100 \text { citations from key database (please specify: } \\
\text { team to determine what additional Subject Headings and text words are needed }\end{array}$ \\
\hline$\square$ & Search trials registry (if required) \\
\hline$\square$ & Provide files in citation manager compatible format of all retrieved publications \\
\hline$\square$ & Provide a PRISMA flowchart template with initial results \\
\hline$\square$ & Perform cited reference search of eligible studies (if required) \\
\hline$\square$ & $\begin{array}{l}\text { Prior to the final write-up, rerun the key database search strategy to retrieve newly } \\
\text { published information }\end{array}$ \\
\hline$\square$ & Write the "sources and search method" section \\
\hline$\square$ & Attend team meetings, if required (to offer training and respond to workflow issues) \\
\hline$\square$ & $\begin{array}{l}\text { Submit strategy for peer review (if required) -- in-house or through PRESS (no } \\
\text { guarantee strategy will be selected for review) - please note that this will add 2-3 } \\
\text { weeks to review timelines }\end{array}$ \\
\hline$\square$ & Provide Endnote training and support \\
\hline$\square$ & Other: \\
\hline
\end{tabular}

You and Your Team will:

\begin{tabular}{|c|c|}
\hline$\square$ & Write review protocol \\
\hline$\square$ & Register protocol with PROSPERO, or submit for publication (as appropriate) \\
\hline$\square$ & Keep records of decisions and data (to compile the PRISMA flow diagram) \\
\hline$\square$ & Search reference lists of eligible studies \\
\hline$\square$ & Include UHN IS as co-author on any output from this review* \\
\hline$\square$ & $\begin{array}{l}\text { Provide manuscript to Information Specialist for review and feedback prior to } \\
\text { submission }\end{array}$ \\
\hline$\square$ & Other: \\
\hline
\end{tabular}

* Your UHN IS is entitled to co-authorship according to the UHN Research Authorship Policy 40.60.001 and the International Committee of Medical Journal Editors' Recommendations for the Conduct, Reporting, Editing, and Publication of Scholarly Work in Medical Journals, and reserves the right to final approval of any version sent for publication. 
Yang, Orchanian-Cheff, Anderson, Farrell, Tripp

Optional Fee-based Services

Please discuss these fees with your IS. Our fee guide is available online.

\begin{tabular}{ll}
$\begin{array}{c}\text { Library } \\
\text { Services }\end{array}$ & $\begin{array}{c}\text { The SR } \\
\text { team }\end{array}$ \\
\hline$\square$ & $\begin{array}{l}\text { Search grey literature } \\
\text { Remove duplicate citations } \\
\square\end{array}$ \\
$\square$ & $\begin{array}{l}\text { Hand search selected journals (number: } \\
\square\end{array}$ \\
$\square$ & $\begin{array}{l}\text { Pearch conferences individually } \\
\square\end{array}$
\end{tabular}

\section{Estimated Library Service Costs:}

- $\quad$ To be filled out by the IS based on information provided in the project proposal.

- $\quad$ Please note that these costs are simply estimates and actual costs may vary. 
Yang, Orchanian-Cheff, Anderson, Farrell, Tripp

\section{AGREEMENT}

I understand that:

(1) UHN Information Specialists co-authors have the right to final approval of any version sent for publication.

(2) Library and Information Services reserves the right to withdraw involvement in any project that veers away from Best Practices.

(3) Charges will apply for any fee-based services rendered.

(4) Estimated service costs are estimates only, and actual costs may vary.

As Principal Investigator I agree to:

(1) Grant co-authorship in accordance with the UHN Research Authorship Policy 40.60 .001 and the International Committee of Medical Journal Editors' Recommendations for the Conduct, Reporting, Editing, and Publication of Scholarly Work in Medical Journals.

(2) Reimburse UHN Library for any fee-based services provided in accordance with these Terms of Service.

(3) If making an application for a grant or funding, include the costs associated with expert library services and resources in my application, and provide the library with the final decision of the granting body.

\section{UHN Information Specialist}

Name

Signature

Date

\section{Principal Investigator}

Name

\section{Department}

*FCC/SAR \#

Signature

Date

*Any applicable costs will be charged to this FCC/SAR \#. 
Yang, Orchanian-Cheff, Anderson, Farrell, Tripp

\section{PART 5: GLOSSARY OF TERMS}

Literature Review: Generic term: published materials that provide examination of recent or current literature. Can cover wide range of subjects at various levels of completeness and comprehensiveness. May include research findings.

Traditional (narrative) reviews: provide a broad overview of a research topic with no clear methodological approach. Information is collected and interpreted unsystematically with subjective summaries of findings. Authors aim to describe and discuss the literature from a contextual or theoretical point of view. Although the reviews may be conducted by topic experts, due to preconceived ideas or conclusions, they could be subject to bias.

Knowledge Synthesis: “...the contextualization and integration of research findings of individual research studies within the larger body of knowledge on the topic. A synthesis must be reproducible and transparent in its methods, using quantitative and/or qualitative methods. It could take the form of a systematic review, follow the methods developed by the Cochrane Collaboration, result from a consensus conference or expert panel or synthesize qualitative or quantitative results. Realist syntheses, narrative syntheses, meta-analyses, meta-syntheses and practice guidelines are all forms of synthesis." Canadian Institutes of Health Research. (2016, July 28). Knowledge Translation. Retrieved April 26, 2018, from http://www.cihrirsc.gc.ca/e/29418.html

Systematic Review: "A systematic review attempts to identify, appraise and synthesize all the empirical evidence that meets pre-specified eligibility criteria to answer a given research question. Researchers conducting systematic reviews use explicit methods aimed at minimizing bias, in order to produce more reliable findings that can be used to inform decision making." (See Section 1.2 in the Cochrane Handbook for Systematic Reviews of Interventions.) May or may not include meta-analysis.

Meta-Analysis: A subset of systematic reviews; Technique that statistically combines the results of quantitative studies to provide a more precise effect of the results.

Scoping Review: "A scoping review or scoping study is a form of knowledge synthesis that addresses an exploratory research question aimed at mapping key concepts, types of evidence, and gaps in research related to a defined area or field by systematically searching, selecting and synthesizing existing knowledge." Colquhoun, H. L., Levac, D., O'Brien, K. K., Straus, S., Tricco, A. C., Perrier, L., ... Moher, D. (2014). Scoping reviews: Time for clarity in definition, methods, and reporting. Journal of Clinical Epidemiology, (12), 1291-1294

Rapid Review: "...a type of knowledge synthesis in which systematic review processes are accelerated and methods are streamlined to complete the review more quickly than is the case for typical systematic reviews. Rapid reviews take an average of 5-12 weeks to complete, thus providing evidence within a shorter time frame required for some health policy and systems decisions." Tricco AC, Langlois EV, Straus SE, editors. Rapid reviews to strengthen health policy and systems: a practical guide. Geneva: World Health Organization; 2017. Licence: CC BY-NC-SA 3.0 IGO. 
Yang, Orchanian-Cheff, Anderson, Farrell, Tripp

\section{Appendix 2: Interview Guide}

*Ask if okay with audio recording

*Inform some questions may be obvious

1. When did you start working for UHN as an information specialist?

2. How would you describe your role as an information specialist?

3. How do you participate in knowledge syntheses?

4. What is a systematic review, and what is involved in doing one?

5. Who are your clients? What do they want from you in terms of systematic reviews?

6. What is involved in the preparation for a systematic review?

7. What is involved in the follow-up process of a systematic review?

8. How was your experience with the former knowledge synthesis service model?

9. What worked in that service model? What didn't?

10. What prompted the change to the new service model?

11. Systematic reviews seem to be taking more and more time. Why do you think this is happening?

12. Has the process of doing systematic reviews changed over time? How so? Why?

13. How was the new service model designed?

14. Was the design based on another service model? If so, what was it?

15. Was the design influenced by the any literature? If so, which sources?

16. Who was involved in the design? How were they involved? 
Yang, Orchanian-Cheff, Anderson, Farrell, Tripp

17. What are your thoughts and opinions regarding the current knowledge synthesis service model?

18. What changes have you observed since the implementation of the new service model?

19. What do you think still needs to be improved?

20. How have the changes impacted the quality of your systematic reviews or other IS work?

21. Were there any challenges in transitioning to the new service model? 